\title{
ONE HUNDRED YEARS SINCE THE INTRODUCTION OF THE SET DISTANCE BY DIMITRIE POMPEIU
}

\author{
T. Birsan ${ }^{1}$ and D. Tiba ${ }^{2}$ \\ 1 "Gh. Asachi" University, Department of Mathematics, Iasi, Romania, tbi@math.tuiasi.ro \\ ${ }^{2}$ Institute of Mathematics, Romanian Academy, Bucharest, Romania, dan.tiba@imar.ro
}

\begin{abstract}
This paper recalls the work of D. Pompeiu who introduced the notion of set distance in his thesis published one century ago. The notion was further studied by F. Hausdorff, C. Kuratowski who acknowledged in their books the contribution of Pompeiu and it is frequently called the Hausdorff distance.
\end{abstract}

keywords: Hausdorff distance, Hausdorff-Pompeiu distance, Pompeiu functions, Pompeiu conjecture, Schiffer conjecture

\section{The thesis of Pompeiu}

On March 31-st, 1905, Dimitrie Pompeiu (1873-1954), a distinguished Romanian mathematician, defended at the Faculté de Sciences de Paris his Ph.D. work Sur la continuité des fonctions de variables complexes. The comission was chaired by H. Poincaré and included G. Koenigs and E. Goursat and the work was published the same year in [14]. P. Montel, a wellknown French mathematician, has appreciated the remarkable contribution of Pompeiu's thesis by the words: Pour un coup d'essai, c'est un coup de maître, [1]. Ideas, notions and results discussed in the thesis of Pompeiu have attracted the interest of important contemporary mathematicians: P. Painlevé, A. Denjoy, F. Hausdorff and is now a part of the universal mathematical heritage. Pompeiu studied a problem formulated by Painlevé [13] already in 1897, concerning the singularities of uniform analytic functions. At that time, it was generally admitted that a uniform analytic function cannot be continuously extended on the set of its singularities. However Pompeiu could construct examples of such functions which are continuous on the set of their singularities and this set has positive measure. The controversy that followed was solved in 1909 by Denjoy who confirmed that the arguments of Pompeiu are correct. This was a turning point in the theory of uniform analytic functions and Pompeiu was considered as the best specialist in this field, at the beginning of the last century [1]. Pom-

Please use the following format when citing this chapter:

Author(s) [insert Last name, First-name initial(s)], 2006, in IFIP International Federation for Information Processing, Volume 199, System Modeling and Optimization, eds. Ceragioli F, Dontchev A., Furuta H., Marti K., Pandolfi L., (Boston: Springer), pp. [insert page numbers]. 

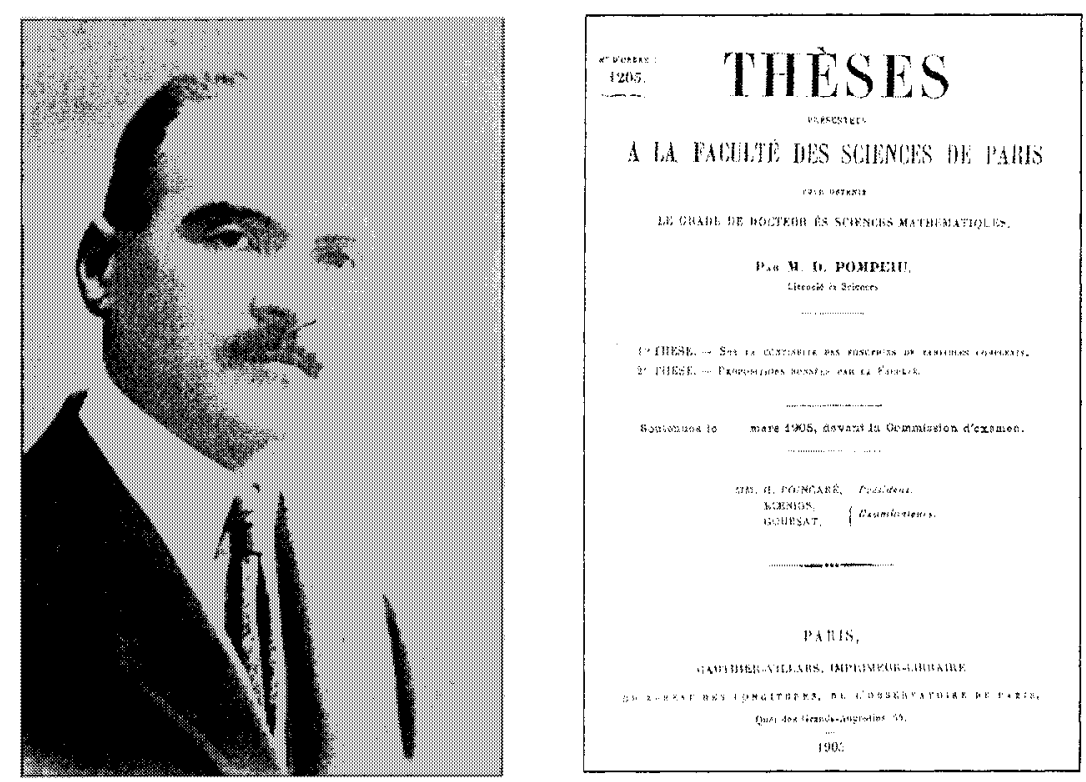

peiu used an example due to A. Koepcke (pag.45 in [14]) which was rather complicated. Some years later, in [16], he constructed much simpler examples of the same type, that is real functions with bounded derivatives that have roots in any interval and are not identically zero in some interval. Such functions are now called Pompeiu functions and are included in many monographs on real functions.

Very well documented presentations of this moment from the history of mathematics may be found in [8], [9]. In his arguments in the complex plane, Pompeiu needed a notion of distance between closed curves. It is to be remarked that quite in the same period, M. Fréchet introduced in his Ph.D. thesis (published a little bit later in [3]) the distance between two elements and the axioms of metric spaces. This is the context in which D. Pompeiu defines in 1905 the notions of écart and écart mutuel between two sets ([14], p.17 and 18). Preserving his notations, let $E_{h}$ and $E_{k}$ be two compact subsets in the plane. The écart of $E_{h}$ with respect to $E_{k}$, denoted by $\Delta_{h k}$ was defined as the maximum of the distances of an arbitrary point $P_{h} \in E_{h}$ to the set $E_{k}$. The sum $\Delta_{h k}+\Delta_{k h}$ was called the reciprocal distance (écart mutuel) between the sets $E_{h}$ and $E_{k}$. This allows Pompeiu to see the compact subsets in the plane as the elements of another set and to define in a natural way limits, closure, etc. for this "set of sets". Consequently, Pompeiu is also considered as one of the founders of the theory of hyperspaces, see McAllister [10]. The thesis of D. Pompeiu is the birth certificate of the notion of distance between sets and its importance 
was immediately remarked by the contemporary mathematicians. Already in 1905, in his report for the Jahrbuch fuer Mathematik, on p.455 A. Gutzmer calls the Pompeiu distance Einfuehrung eines neuen Begriffes der Mengenlehre (the introduction of a new concept in set theory). In 1914,in his famous book [5], Hausdorff studies the notion of set distance, in the natural setting of metric spaces and with a small modification (the sum si replaced by the maximum) :

$$
d(A, B)=\max \left\{\sup _{x \in A} d(x, B), \sup _{y \in B} d(y, A)\right\} .
$$

He devotes a consistent paragraph to the study of the properties of the distance and he quotes correctly Pompeiu [5] on p. 463, as the author of this notion. Moreover he also establishes that the distances defined with the "sum" or with the "max" give the same toplogy, that is they are equivalent. The same considerations may be found in his book from 1927, [6], where Pompeiu is quoted at page 280 . It is to be noted that at that time, the main text of a book did not include any reference to the origin of a subject and all these references were concentrated in a short appendix at the end (Quellenangabe). Probably, due to this reason, many mathematicians didn't notice the reference to Pompeiu's work [14] and the set distance is frequently termed as the Hausdorff distance. Other names used in the scientific literature are Pompeiu distance or Pompeiu-Hausdorff distance, Hausdorff-Pompeiu distance. The great Polish mathematician C. Kuratowski, in his treatise [7], on p. 106 mentions both the thesis of Pompeiu from 1905 and the book of Hausdorff from 1914. This is done in a footnote, on the page where the notion is introduced and is completely accessible to the reader. In 1978, McAllister [10] published a remarkable historical study devoted to the first 50 years in the theory of hyperspaces. The role of Pompeiu's thesis is underlined clearly: "who [Pompeiu] may with some justice be said to have invented hyperspaces, and Hausdorff's use of them in 1914 in his treatise Grundzuege der Mengenlehre had made them very well known" (p. 310) and similarly on p. 311 "I have found no evidence of the Hausdorff metric itself before Pompeiu's thesis". There are many notions of set distance which are not equivalent with the Hausdorff-Pompeiu distance. For instance, one can define the distance between two Lebesgue measurable subsets in an Euclidean space as the measure of their symmetric difference. However, the Hausdorff-Pompeiu metric seems to be the only one with a remarkable compactness property: if $\left(A_{n}\right)$ is a sequence of compacts, bounded with respect to $n$, then there is a compact subset $\mathrm{A}$ and a subsequence again indexed by $n$, such that :

$$
\lim _{n \rightarrow \infty} d\left(A_{n}, A\right)=0 .
$$

This property makes the Hausdorff-Pompeiu distance a fundamental notion in the study of the topologies on families of subsets and in the modern theory 
of shape optimization (optimal design). The interested reader may consult the recent monograph [11] and its references for a survey of the mathematical literature on geometric optimization.

\section{The Pompeiu conjecture}

The whole mathematical work of Pompeiu is characterized by a profound originality, by the introduction of many fruitful ideas and methods. We brieffy recall the definition in 1912 of the areolar derivative (see [15]), further developed by M. Nicolescu, Gh. Calugareanu, N. Cioranescu, N. Teodorescu, Gr. Moisil and other. This is in fact the fundamental $\bar{\partial}$ - operator from complex analysis. In this context, Pompeiu also proved the Cauchy-Pompeiu formula which appeared here for the first time, [9]. Probably the best known paper of Pompeiu is his Note [17] from 1929 (recent estimates indicate almost one thousand articles quoting it). Here one can find the famous Pompeiu conjecture, still unsolved completely :

Let $f$ be a continuous function in the plane and $D$ a compact subset such that

$$
\int_{\sigma(D)} f(x, y) d x d y=0
$$

where $\sigma(D)$ denotes any compact subset in the plane obtained by rigid motions of $D$. Then, is it true that $f$ is null in the plane?

For the case when $D$ is a disk, there are counterexamples [4] of the form $f(x, y)=\sin (a x+b y)$ with $a, b$ appropriately chosen real numbers. For any other domains in the plane, the answer seems to be positive although just some special cases are solved [19]. We underline the fundamental character in the mathematical analysis of this property (if proved) and its relationship with the Schiffer conjecture (formulated later) concerning the eigenvalues of the Laplace operator with Cauchy conditions on the boundary, [2], [18].

O. Onicescu, a student of Pompeiu and a wellknown Romanian mathematician, said that the creations of Dimitrie Pompeiu are "simple, plastic, global and full of significance in the world of science", [12].

\section{References}

[1] G. Andonie. Istoria Matematicii in Romania. Ed. Ştiinţifică, Bucharest, 1965.

[2] C.A. Bernstein. An inverse spectral theorem and its relation to the Pompeiu problem. $J$. d'Analyse Math. 37:128-144, 1980.

[3] M. Fréchet. Sur quelques points du calcul fonctionnel (Thèse). Rend. Circ. Mat. Palermo. 22:1-74, 1906 
[4] N. Garofalo, F. Segala. Univalent functions and the Pompeiu problem. Trans. A.M.S. 346:137-146, 1994

[5] F. Hausdorff. Grundzuege der Mengenlehre, Viet, Leipzig, 1914.

[6] F. Hausdorff. Mengenlehre. Walter de Gruyter, Berlin, 1927.

[7] C. Kuratowski. Topologie I. Polish Math. Soc., Warsaw, 1952.

[8] S. Marcus. Funcţiile lui Pompeiu. Studii şi Cerc. Mat. 5:413-419, 1954.

[9] M. Mitrea, F. Şabac. Pompeiu's integral representation formula. History and mathematics. Rev.Roum.Math.Pures Appl. 43:211-226, 1998.

[10] B.L. McAllister. Hyperspaces and multifunctions, the first halfcentury (1900-1950). Nieuw Arch. Wisk. 26:309-329, 1978.

[11] P. Neittaanmaki, J. Sprekels, D. Tiba. Optimization of elliptic systems.Theory and applications. Springer Verlag, New York, 2005.

[12] O. Onicescu. Pe drumurile vieții. Ed. Şt. şi Enciclopedică, Bucharest, 1981.

[13] P. Painlevé. Leçons sur la théorie analytique des équations differentielles, professées a Stockholm. Hermann, Paris, 1897.

[14] D. Pompeiu. Sur la continuité des fonctions de variables complexes (Thèse). GauthierVillars, Paris, 1905; Ann.Fac.Sci.de Toulouse 7:264-315, 1905.

[15] D. Pompeiu. Opera Matematică. Ed.Acad.Române, Bucharest, 1959.

[16] D. Pompeiu. Sur les fonctions derivées. Math.Ann. 63:326-332, 1907.

[17] D. Pompeiu. Sur certains systemes d'équations linéires et sur une propriété intégrale des fonctions de plusieurs variables. C.R.Acad.Sc.Paris. 188:1138-1139, 1929.

[18] M. Vogelius. An inverse problem for the equation $\Delta u=-c u-d$. Annales de l'Institut Fourier. 44:1181-1209, 1994.

[19] S.A. Williams. A partial solution of the Pompeiu problem. Math. Ann. 223;183-190, 1976. 